\title{
ON BERNSTEIN INEQUALITY FOR VECTORS IN BANACH SPACES
}

\section{E.E. DIKAREV}

\begin{abstract}
We obtain the Bernstein inequality for the vectors in the Banach space of the isometric representation of a one-parametric group of the operators. We introduce the notion of an entire at infinity function. For such functions and for the norms of commutation operators we obtain the Bernstein inequality.
\end{abstract}

Keywords: Banach modulus, isometric representation, Beurling spectrum, entire function, commutation operator.

Mathematics Subject Classification: 47A10, 47L10

\section{INTRODUCTION}

Consider the Banach space $\mathrm{C}_{2 \pi}(\mathbb{R})$ of continuous $2 \pi$-periodic complex-valued functions defined on $\mathbb{R}$ as well as trigonometric polynomials $x \in \mathrm{C}_{2 \pi}(\mathbb{R})$ of the form

$$
x(t)=\sum_{k=-n}^{n} \alpha_{k} \mathrm{e}^{\mathrm{i} k t}, \quad\left|\alpha_{-n}\right|+\left|\alpha_{n}\right|>0 .
$$

S.N. Bernstein obtained the following inequality

$$
\max _{t \in[0,2 \pi]}\left|x^{\prime}(t)\right| \leqslant n \cdot \max _{t \in[0,2 \pi]}|x(t)| .
$$

It was generalized in various directions. For instance, Bernstein inequality was obtained for an entire function of exponential type $\sigma$ bounded on the real axis (see [1]):

$$
\sup _{t \in \mathbb{R}}\left|x^{\prime}(t)\right| \leqslant \sigma \cdot \sup _{t \in \mathbb{R}}|x(t)| .
$$

Let $\mathscr{X}$ be a complex Banach space. By End $\mathscr{X}$ we denote the Banach algebra of linear bounded operators acting in $\mathscr{X}$.

We shall call a closed linear operator $A: \mathrm{D}(A) \subset \mathscr{X} \rightarrow \mathscr{X}$ correct (see [2]) (or self-adjoint [3, 4]), if the operator i $A$ is the generator (generating operator) of a strongly continuous group of isometric operators $T: \mathbb{R} \rightarrow$ End $\mathscr{X}$.

In particular, in the classical Bernstein theorem operator $A$ is defined as $A=\mathrm{i}^{-1} \frac{\mathrm{d}}{\mathrm{d} t}=-\mathrm{i} \frac{\mathrm{d}}{\mathrm{d} t}$, it acts in the space of uniformly continuous bounded complex-valued functions $\mathrm{C}_{\mathrm{bu}}(\mathbb{R})$ and is the generator of the group of shifts. We observe that the spectrum of the operator obeys $\sigma(A) \subset \mathbb{R}$ (see [3]). In paper by A.G. Baskakov [5] it was proven for a bounded correct operator in a Banach space that $\|A\|=r(A)$, where $r(A)$ is the spectral radius of operator $A$.

In the present Bernstein inequality is obtained for the vectors in a Banach space, in which an isometric group of operators with the generator i $A$ acts. The generator can be an unbounded operator.

E.E. Dikarev, On Bernstein inequality for vectors in Banach spaces.

(c) E.E. DikAREV. 2013.

THE WORK IS SUPPORTED BY RFBR (GRANT NO. 13-01-00378).

Submitted September 21, 2013. 
We obtain applications of Bernstein inequality for the functions of exponential type at infinity and the estimates for the commutation operator. For such operator we obtain the estimate

$$
\|A x\| \leqslant r(x) \cdot\|x\|, \quad x \in \mathscr{X},
$$

where $r(x)$ is the spectral radius of the vector $x$ which will be defined below.

In particular for $x \in \mathrm{C}_{2 \pi}(\mathbb{R})$ being a trigonometrical polynomial $(1)$ we have $r(x)=n$. Thus, estimate (2) is a direct generalization of Bernstein inequality.

\section{Properties of Beurling spectrum}

Let $\mathrm{L}^{1}(\mathbb{R})$ be the Banach algebra of all summable on $\mathbb{R}$ complex-valued functions with the convolution of functions as the multiplication and with the norm

$$
\|f\|_{1}=\int_{\mathbb{R}}|f(t)| \mathrm{d} t, \quad f \in \mathrm{L}^{1}(\mathbb{R}),
$$

and let $T: \mathbb{R} \rightarrow$ End $\mathscr{X}$, where $\mathscr{X}$ is a Banach space, be a strongly isometric representation. Then $\mathscr{X}$ is equipped by the structure of $\mathrm{L}^{1}(\mathbb{R})$-module by the formula

$$
f x=\int_{\mathbb{R}} f(t) T(-t) x \mathrm{~d} t, \quad f \in \mathrm{L}^{1}(\mathbb{R}), \quad x \in \mathscr{X} .
$$

The module structure on $\mathrm{C}_{\mathrm{b}}(\mathbb{R})$ is defined by formula $(3)$ by means of the representation $(T(t) \varphi)(s)=$ $\varphi(s+t), \varphi \in \mathrm{C}_{\mathrm{b}}(\mathbb{R}), t, s \in \mathbb{R}$, i.e., by the usual convolution of functions. In view of formula (3), a Banach $\mathrm{L}^{1}(\mathbb{R})$-module $\mathscr{X}$ will be sometimes denoted as $(\mathscr{X}, T)$.

Definition 1 (See [3, 4, 5]). Beurling spectrum of a vector $x$ in the Banach $\mathrm{L}^{1}(\mathbb{R})$-module $\mathscr{X}$ is the set $\Lambda(x)$ in $\mathbb{R}$ being the complement in $\mathbb{R}$ of set $\left\{\lambda_{0} \in \mathbb{R} \mid\right.$ there exists a function $f_{0} \in$ $\mathrm{L}^{1}(\mathbb{R})$ such that $\widehat{f_{0}}\left(\lambda_{0}\right) \neq 0$ and $\left.f_{0} x=0\right\}$.

Example 1. The Beurling spectrum of a trigonometrical polynomial (1) is the set of $k$ satisfying $\alpha_{k} \neq 0$.

Definition 2. Let $M$ be an arbitrary subset in Banach module $\mathscr{X}$. Beurling spectrum of set $M$ is the set $\Lambda(M)$ in $\mathbb{R}$ being the complement in $\mathbb{R}$ of the set $\left\{\lambda_{0} \in \mathbb{R} \mid\right.$ there exists $f_{0} \in$ $\mathrm{L}^{1}(\mathbb{R})$ such that $\widehat{f}_{0}\left(\lambda_{0}\right) \neq 0$ and $f_{0} x=0$ for each $\left.x \in M\right\}$.

Lemma 1. The following properties of Beurling spectrum for vectors in Banach $\mathrm{L}^{1}(\mathbb{R})$-module $(\mathscr{X}, T)$ hold true:

1. $\Lambda(x)$ is a closed subspace in $\mathbb{R}$ and $\Lambda(x)=\varnothing \Leftrightarrow x=0$;

2. $\Lambda(f x) \subset(\operatorname{supp} \widehat{f}) \cap \Lambda(x), f \in \mathrm{L}^{1}(\mathbb{R}), x \in \mathscr{X}$. In particular, if $x$ has a compact Beurling spectrum, then the vector $f x$ has a compact Beurling spectrum as well;

3. $f x=0$ if $\widehat{f}=0$ on set $\Lambda(x)$ and $(\operatorname{supp} \widehat{f}) \cap \Lambda(x)$ is at most countable;

4. $f x=x$ if set $\Lambda(x)$ is compact and $\widehat{f} \equiv 1$ is the vicinity of set $\Lambda(x)$.

Remark 1 (See [3]). If $f x=0$ for each function $f \in \mathrm{L}^{1}(\mathbb{R})$, it implies $x=0$.

Definition 3. A linear subspace $E$ of $\mathrm{L}^{1}(\mathbb{R})$-module $(\mathscr{X}, T)$ is called submodule, if it is invariant w.r.t. all the operators $T(t), t \in \mathbb{R}$, and $T(f), f \in \mathrm{L}^{1}(\mathbb{R})$.

Let $\Delta$ be a closed set in $\mathbb{R}$. The submodule

$$
\mathscr{X}(\Delta)=\{x \in \mathscr{X} \mid \Lambda(x) \subset \Delta\}
$$

is called spectral submodule.

Lemma 2. Let $\mathscr{X}$ be a Banach $\mathrm{L}^{1}(\mathbb{R})$-module, $\Delta$ be a closed set in $\mathbb{R}$. Then $\mathscr{X}(\Delta)$ is a closed submodule and $\Lambda(\mathscr{X}(\Delta)) \subset \Delta$. 
Lemma 3. Let $f \in \mathrm{L}^{1}(\mathbb{R})$ be so that $\operatorname{supp} \widehat{f}$ is a compact set and $[-a, a], a>0$ is the smallest segment comprising supp $\widehat{f}$. Then $f$ is infinitely differentiable. Moreover, it can be continued to an entire function $\tilde{f}: \mathbb{C} \rightarrow \mathbb{C}$ of exponential type $\leqslant$ a, i.e., the estimate

$$
|\widetilde{f}(z)| \leqslant \frac{1}{2 \pi} \max |\widehat{f}(\lambda)| \cdot \mathrm{e}^{a|z|}, \quad z \in \mathbb{C}
$$

holds true.

Lemma 4. Let $\widehat{f} \equiv 1$ in a vicinity of the set $\Lambda(x)$, where $x$ is an element of a Banach $\mathrm{L}^{1}(\mathbb{R})$-module $\mathscr{X}$. Then the identities

$$
T(t) x=T(t)(f x)=f_{t} x, \quad t \in \mathbb{R}, \quad \text { where } f_{t}(s)=(S(t) f)(s)=f(t+s), \quad s \in \mathbb{R}, \quad x \in \mathscr{X},
$$

hold true.

Proof. The first identity is implied by Property 4 of Lemma 1. Then

$$
\begin{aligned}
T(t)(f x) & =T(t) \int_{\mathbb{R}} f(\tau) T(-\tau) x \mathrm{~d} \tau=\int_{\mathbb{R}} f(\tau) T(t) T(-\tau) x \mathrm{~d} \tau \\
& =\int_{\mathbb{R}} f(\tau) T(t-\tau) x \mathrm{~d} \tau=\int_{\mathbb{R}}(S(t) f)(s) T(-s) x \mathrm{~d} s=f_{t} x .
\end{aligned}
$$

In what follows we make use of the powers $A^{n}: \mathrm{D}\left(A^{n}\right) \subset \mathscr{X} \rightarrow \mathscr{X}$ of operator $A$ which we denote according book [6].

Definition 4. For $n=0,1, \ldots$ the operator $A^{n}$ is defined by the induction by the relations $A^{0}=I$, $A^{1}=A$ and

$$
\begin{aligned}
\mathrm{D}\left(A^{n}\right)= & \left\{x \in \mathscr{X} \mid x \in \mathrm{D}\left(A^{n-1}\right), \quad A^{n-1} x \in \mathrm{D}(A)\right\}, \\
& A^{n} x=A\left(A^{n-1} x\right), \quad x \in \mathrm{D}\left(A^{n}\right) .
\end{aligned}
$$

Lemma 5. Let a vector $x$ in $\mathrm{L}^{1}(\mathbb{R})$-module $(\mathscr{X}, T)$ has a compact Beurling spectrum $\Lambda(x)$ and a function $f \in \mathrm{L}^{1}(\mathbb{R})$ is such that $\widehat{f}(\lambda)=\lambda$ is a neighborhood $\mathrm{U}$ of set $\Lambda(x)$. Then $x \in \mathrm{D}\left(A^{n}\right)$ for each $n \geqslant 1$ and $f x=A x$.

Proof. We choose a function $\varphi \in \mathrm{L}^{1}(\mathbb{R})$ such that $\widehat{\varphi} \equiv 1$ in a neighborhood $\mathrm{V}$ of set $\Lambda(x)$ and $\operatorname{supp} \widehat{\varphi}$ is a compact set. By Lemma 1, $\varphi x=x$. Moreover, we can choose $\varphi$ to satisfy the following properties: $\varphi$ is infinitely differentiable, $\varphi^{\prime} \in \mathrm{L}^{1}(\mathbb{R})$, and $\widehat{\varphi^{\prime}}(\lambda)=\mathrm{i} \lambda \widehat{\varphi}(\lambda)$. Consider the function $\psi=-\mathrm{i} \varphi, \psi \in \mathrm{L}^{1}(\mathbb{R})$. Therefore, $\widehat{\psi}^{\prime}(\lambda)=\lambda \widehat{\varphi^{\prime}}(\lambda)$. Moreover, $f-\psi \in \mathrm{L}^{1}(\mathbb{R})$, and $\widehat{f}-\widehat{\psi}^{\prime}=0$ in the neighborhood $\mathrm{U} \cap \mathrm{V}$ of set $\Lambda(x)$. Therefore, by Property 2 of Lemma 1, $\left(f-\psi^{\prime}\right) x=0$ that implies $f x=\psi^{\prime} x$. Thus, it is sufficient to prove the lemma for function $\psi^{\prime}$.

Since function $\varphi$ satisfies the hypothesis of Lemma 4 , the identities

$$
\lim _{t \rightarrow 0} \frac{T(t) x-x}{t}=\lim _{t \rightarrow 0} \frac{T(t)(\varphi x)-\varphi x}{t}=\lim _{t \rightarrow 0} \frac{\varphi_{t} x-\varphi x}{t}=\lim _{t \rightarrow 0} \frac{\left(\varphi_{t}-\varphi\right) x}{t}=\varphi^{\prime} x=\mathrm{i} \psi^{\prime} x=\mathrm{i} A x
$$

hold true. Thus, $x \in \mathrm{D}(A)$. Moreover, the identity $\varphi^{\prime} x=\mathrm{i} A x$ yields $\psi^{\prime} x=A x$, or $f x=A x$.

The obtained representation $A x=f x$ and Property 2 of Lemma 1 imply that the vector $f x$ has a compact Beurling spectrum and thus by the proven above $f x=A x \in \mathrm{D}(A)$, at that, $A^{2} x=f A x$. It follows from the definition of $A^{n}$ that $x \in \mathrm{D}\left(A^{n}\right)$ for each $n \geqslant 1$.

Theorem 1. If $x \in \mathscr{X}$ has a compact Beurling spectrum, then $x \in \mathrm{D}\left(A^{m}\right)$ for each $m \in \mathbb{N}$, $\Lambda\left(A^{m} x\right) \subset \Lambda(x)$ and the estimates

$$
\left\|A^{m} x\right\| \leqslant r(x)^{m} \cdot\|x\|
$$

hold true for each $m \geqslant 1$. 
Proof. The fact $x \in \mathrm{D}\left(A^{m}\right)$ follows from Lemma 5. Let us show that $\Lambda\left(A^{m} x\right) \subset \Lambda(x)$. Assume that $\lambda_{0} \notin \Lambda(x)$. Then by the definition there exists a function $f_{0} \in \mathrm{L}^{1}(\mathbb{R})$ such that $\widehat{f}_{0}\left(\lambda_{0}\right) \neq 0$ and $f_{0} x=0$. Then $f_{0}(A x)=A f_{0} x=0$ and it implies

$$
\Lambda(A x) \subset \Lambda(x), \quad \Lambda\left(A^{n} x\right) \subset \Lambda(x) .
$$

Consider the vector $y$ of the form

$$
y=r(x) \sum_{k=-\infty}^{\infty} \frac{T\left(\frac{k \pi-\pi}{r(x)}\right) x}{(\pi / 2-k \pi)^{2}}
$$

Let a function $\psi \in \mathrm{L}^{1}(\mathbb{R})$ be so that $\widehat{\psi}(\lambda)=\lambda$ in a vicinity of set $\Lambda(x)$. Then by Lemma 5 the identity $A x=\psi x$ holds true. By Lemma 4 , for each function $f \in \mathrm{L}^{1}(\mathbb{R})$ we obtain the identities

$$
f(y-A x)=f(y-\psi x)=r(x) \cdot\left(\sum_{k=-\infty}^{\infty} \frac{f_{\frac{k \pi-\pi}{r(x)}}}{(\pi / 2-k \pi)^{2}}\right) x-(\psi * f) x=g x-(\psi * f) x=\varphi x,
$$

where the Fourier transform of $g \in \mathrm{L}^{1}(\mathbb{R})$ reads as

$$
\widehat{g}(\lambda)=r(x) \sum_{k=-\infty}^{\infty} \frac{\widehat{f}(\lambda) \mathrm{e}^{i \frac{k \pi-\pi}{r(x)} \lambda}}{(\pi / 2-k \pi)^{2}}
$$

and $\varphi=g-\psi * f$. Since $x$ has a compact Beurling spectrum, we have $r(x)<\infty$. The series in the right hand side of (5) converges absolutely. Function $\varphi$ satisfies the hypothesis of Property 3 in Lemma 1. Thus, $f(y-A x)=0$ for each function $f \in \mathrm{L}^{1}(\mathbb{R})$, and this is why $y-A x=0$ by Remark 1 . The estimates

$$
\|A x\|=r(x) \cdot\left\|\sum_{k=-\infty}^{\infty} \frac{T\left(\frac{k \pi-\pi}{r(x)}\right) x}{(\pi / 2-k \pi)^{2}}\right\| \leqslant r(x) \sum_{k=-\infty}^{\infty} \frac{1}{(\pi / 2-k \pi)^{2}} \cdot\|x\|=r(x) \cdot\|x\|
$$

hold true. Inclusion (4) and the proven above yield

$$
\left\|A^{n} x\right\|=\left\|A^{n-1} A x\right\| \leqslant r(x)^{n}\|x\|, \quad n \geqslant 2 .
$$

\section{Bernstein INEQUALITY FOR ENTIRE AT INFINITY FUNCTION}

By $\mathrm{C}_{\mathrm{bu}}(\mathbb{R})$ we denote a closed subspace of uniformly continuous bounded complex-valued functions in $\mathrm{C}_{\mathrm{b}}(\mathbb{R})$ and the symbol $\mathrm{C}_{0}(\mathbb{R})$ stands for the closed subspace $\left\{x \in \mathrm{C}_{\mathrm{bu}}(\mathbb{R})\left|\lim _{|t| \rightarrow \infty}\right| x(t) \mid=0\right\}$.

Definition 5. We call a function $x \in \mathrm{C}_{\mathrm{bu}}(\mathbb{R})$ entire at infinity of exponential type $\sigma \geqslant 0$, if for each $\varepsilon>0$ there exists $x_{0} \in \mathrm{C}_{\mathrm{bu}}(\mathbb{R})$ which can be continued to an entire function $\widetilde{x_{0}}: \mathbb{C} \rightarrow \mathbb{C}$ of exponential type $\sigma+\varepsilon$ such that $x(t)=x_{0}(t)+y_{0}(t)$, where $y_{0} \in \mathrm{C}_{0}(\mathbb{R})$.

Lemma 6. Each function $f \in \mathrm{L}^{1}(\mathbb{R})$ obeying supp $\widehat{f} \in[-\sigma, \sigma]$ can be continued to an entire on $\mathbb{C}$ function of exponential type a. Moreover, for each $z \in \mathbb{C}$, the function $f_{z}(s)=f(s+z), s \in \mathbb{R}$, belongs to algebra $\mathrm{L}^{1}(\mathbb{R})$ and the function $F: \mathbb{C} \rightarrow \mathrm{L}^{1}(\mathbb{R}), F(z)=f_{z}$ is entire of exponential type $\sigma$.

Proof. Let us show that $f$ can be continued to a function of exponential type $\sigma$. We write function $f$ as

$$
f(t)=\frac{1}{2 \pi} \int_{\mathbb{R}} \widehat{f}(\lambda) \mathrm{e}^{\mathrm{i} \lambda t} \mathrm{~d} \lambda=\frac{1}{2 \pi} \int_{-\sigma}^{\sigma} \widehat{f}(\lambda) \mathrm{e}^{\mathrm{i} \lambda t} \mathrm{~d} \lambda
$$

and let

$$
f(z)=\frac{1}{2 \pi} \int_{-\sigma}^{\sigma} \widehat{f}(\lambda) \mathrm{e}^{\mathrm{i} \lambda z} \mathrm{~d} \lambda, \quad z \in \mathbb{C} .
$$


The estimate

$$
|f(z)| \leqslant \frac{1}{2 \pi} 2 \sigma \cdot \max _{\lambda \in[-\sigma, \sigma]}|\widehat{f}(\lambda)| \cdot \mathrm{e}^{\sigma|z|}
$$

is valid. Thus, we have obtained the continuation of function $f$ to an entire on $\mathbb{C}$ function of exponential type $\sigma$. Since $\left(\frac{\mathrm{d} f_{z}}{\mathrm{~d} z}\right)(s)=\frac{\mathrm{d} f(z-s)}{\mathrm{d} z}, z \in \mathbb{C}, s \in \mathbb{R}$, function $F$ is entire of exponential type $\sigma$.

Lemma 7. Function $x \in \mathrm{C}_{\mathrm{bu}}(\mathbb{R})$ can be continued to a function of exponential type $\sigma$ if and only if $\Lambda(x) \subset[-\sigma, \sigma]$.

Proof. Necessity. Suppose that there exists $\lambda_{0}>\sigma+\delta$ for some $\delta>0$. We choose $f_{0} \in \mathrm{L}^{1}(\mathbb{R})$ so that $\widehat{f}_{0}\left(\lambda_{0}\right) \neq 0$, and let $x_{0}=f_{0} * x$, then $\Lambda\left(x_{0}\right) \subset\left[\lambda_{0}-\delta, \lambda_{0}+\delta\right]$. Consider the function $y_{0}(t)=$ $\left(f_{0} * x\right)(t) \mathrm{e}^{-\mathrm{i} \lambda_{0} t}, \Lambda\left(y_{0}\right) \subset[-\delta, \delta]$. Thus, we have constructed the function $\left(f_{0} * x\right)(z)=y_{0}(z) \mathrm{e}^{i \lambda_{0} z}$ of exponential type $\geqslant \sigma+\delta$.

Sufficiency. Let $\Lambda(x) \subset[-\sigma, \sigma]$. We choose $f \in \mathrm{L}^{1}(\mathbb{R})$ such that $\widehat{f}=1$ in the vicinity of the spectrum and $\operatorname{supp} \widehat{f} \subset[-\sigma-\varepsilon, \sigma+\varepsilon]$ for some $\varepsilon>0$. Then $f * x=x, f$ is the entire function with the properties in Lemma 6. By the same symbol $x$ we denote the continuation of $x$ on $\mathbb{C}$. We let $x(z)=\int_{\mathbb{R}} f(z-s) x(s) \mathrm{d} s$ and have

$$
x(z) \leqslant \int_{\mathbb{R}}|f(z-s)| x(s) \mathrm{d} s \leqslant\left\|f_{z}\right\|_{1} \cdot\|x\|, \quad z \in \mathbb{C}, \quad s \in \mathbb{R} .
$$

Since $\left\|f_{z}\right\|_{1} \leqslant$ Const $\cdot \mathrm{e}^{(\sigma+\varepsilon)|z|}, z \in \mathbb{C}, s \in \mathbb{R}$, then $x$ is a function of exponential type $\leqslant \sigma+\varepsilon$. Since $\varepsilon>0$ is arbitrary, we obtain that $x$ is a function of exponential type $\sigma$.

Lemma 8. A function $x \in \mathrm{C}_{\mathrm{bu}}(\mathbb{R})$ is an entire at infinity function of exponential type $\sigma \geqslant 0$ if and only if $(x-f * x) \in \mathrm{C}_{0}(\mathbb{R})$ for each summable function $f$ with the property $\widehat{f} \equiv 1$ on $[-\sigma, \sigma]$.

Definition 6. A function $x \in \mathrm{C}_{\mathrm{bu}}(\mathbb{R})$ is called slowly varying at infinity, if for each $\alpha \in \mathbb{R}$ the inclusion $S(\alpha) x-x \in \mathrm{C}_{0}(\mathbb{R})$ is valid, or, in other words, for each $\alpha \in \mathbb{R}$ the identity

$$
\lim _{|t| \rightarrow \infty}|x(t+\alpha)-x(t)|=0
$$

holds true.

The set of all slowly varying at infinity functions in $\mathrm{C}_{\mathrm{bu}}(\mathbb{R})$ will be indicated by $\mathrm{C}_{\mathrm{sl}}(\mathbb{R})$.

We note that each slowly varying at infinity function is equivalent to an entire at infinity function of exponential type 0 (see [7]).

Consider the strongly continuous group of shifts $S: \mathbb{R} \rightarrow$ End $_{\mathrm{bu}}(\mathbb{R})$ acting by the rule $(S(t) x)(s)=$ $x(s+t), \quad s, t \in \mathbb{R}$. By $\widetilde{S}: \mathbb{R} \rightarrow$ End $\mathrm{C}_{\mathrm{bu}}(\mathbb{R}) / \mathrm{C}_{0}$ we denote the representation defined by the rule $\widetilde{S}(t) \widetilde{x}=\widetilde{S(t) x}$. The module structure is defined by the formula

$$
f \widetilde{x}=\int_{\mathbb{R}} f(t) \widetilde{S}(-t) \widetilde{x} \mathrm{~d} t, \quad f \in \mathrm{L}^{1}(\mathbb{R}), \widetilde{x} \in \mathrm{C}_{\text {bu }}(\mathbb{R}) .
$$

Let $x \in \mathrm{C}_{0}(\mathbb{R})$ and $y \in \mathrm{C}_{\mathrm{bu}}(\mathbb{R})$ is an element of the class $\widetilde{y}$. We introduce the norm of the class of $\widetilde{y}$ by the rule

$$
\|\widetilde{y}\|=\inf _{x \in \mathrm{C}_{0}(\mathbb{R})}\|y+x\| .
$$

Theorem 2. A function $x$ is entire at infinity of exponential type of order $\sigma \geqslant 0$, if $\Lambda(\widetilde{x}) \subset[-\sigma, \sigma]$, or, what is equivalent, if in the class $\widetilde{x}$ there exists a differentiable function $x_{0}$ satisfying the estimate $\left\|x_{0}^{\prime}\right\| \leqslant \sigma\|\widetilde{x}\|$.

The proof follows from the above results and Theorem 1 .

Here we employ the terminology and the results of papers [8, 9, 10]. Let $\mathscr{X}_{1}, \mathscr{X}_{2}$ be Banach spaces and let i $A_{1}, \mathrm{i} A_{2}, A_{k} \in$ End $\mathscr{X}_{k}, k=1,2$, are the generators of isometric groups $T_{1}(t)$ and $T_{2}(t)$, respectively,

$$
T_{1}: \mathbb{R} \rightarrow \text { End } \mathscr{X}_{1}, \quad T_{2}: \mathbb{R} \rightarrow \text { End } \mathscr{X}_{2} .
$$


The Banach space Hom $\left(\mathscr{X}_{1}, \mathscr{X}_{2}\right)$ is equipped by the structure of Banach module by the representation $T: \mathbb{R} \rightarrow$ End $\operatorname{Hom}\left(\mathscr{X}_{1}, \mathscr{X}_{2}\right)$ of the form $T(t) X=T_{2}(t) X T_{1}(-t), t \in \mathbb{R}, X \in \operatorname{Hom}\left(\mathscr{X}_{1}, \mathscr{X}_{2}\right)$. The module structure is defined by means of the formula

$$
(f X) x=\int_{\mathbb{R}} f(\tau)(T(t) X) x \mathrm{~d} \tau=\int_{\mathbb{R}} f(\tau)\left(T_{2}(t) X T_{1}(-t)\right) x \mathrm{~d} \tau .
$$

We note that the representation $T$ is continuous in the strong operator topology.

We denote by the symbol $\operatorname{ad}_{A_{1}, A_{2}}$ the operator $\operatorname{ad}_{A_{1}, A_{2}} X=A_{2} X-X A_{1}$. In the case $A_{1}=A_{2}=A$, $\operatorname{ad}_{A_{1}, A_{2}} X=\operatorname{ad}_{A} X=A X-X A$ is the commutator. Operator $X$ belongs to $\mathrm{D}\left(\operatorname{ad}_{A_{1}, A_{2}}\right)$, if $X \mathrm{D}\left(A_{1}\right) \subset$ $\mathrm{D}\left(A_{2}\right)$, and the operator $A_{2} X-X A_{1}$ has a bounded extension on $\mathscr{X}_{1}$. In what follows we shall denote this extension by the same symbol $A_{2} X-X A_{1}$. We note that the spectrum of the operator $\operatorname{ad}_{A_{1}, A_{2}}$ is the set

$$
\sigma\left(\operatorname{ad}_{A_{1}, A_{2}}\right)=\overline{\left\{\lambda_{2}-\lambda_{1}: \lambda_{1} \in \sigma\left(A_{1}\right), \lambda_{2} \in \sigma\left(A_{2}\right)\right\}},
$$

where $\sigma\left(A_{1}\right), \sigma\left(A_{2}\right)$ are respectively the spectra of the operators $A_{1}, A_{2}$.

Lemma 9. If $X \in \operatorname{Hom}\left(\mathscr{X}_{1}, \mathscr{X}_{2}\right)$ has a compact Beurling spectrum, then $X \in \mathrm{D}\left(\operatorname{ad}_{A_{1}, A_{2}}\right)$, i.e., the operator $A_{2} X--X A_{1} \in \operatorname{Hom}\left(\mathscr{X}_{1}, \mathscr{X}_{2}\right)$ is correct.

Theorem 3. If the Beurling spectrum $\Lambda(X, T)$ of an operator $X \in \operatorname{Hom}\left(\mathscr{X}_{1}, \mathscr{X}_{2}\right)$ is a compact set, then the inequality

$$
\left\|A_{2} X-X A_{1}\right\| \leqslant r(X) \cdot\|X\|
$$

holds true.

The proof follows from Theorem 1 and the above results.

Let $\mathscr{X}_{1}, \mathscr{X}_{2}$ be separable infinite-dimensional space with unconditional bases $\left(e_{k}^{\prime}\right)$ and $\left(e_{k}^{\prime \prime}\right)$, respectively. The matrix $\left(x_{m n}\right)$ of the operator $X \in \operatorname{Hom}\left(\mathscr{X}_{1}, \mathscr{X}_{2}\right)$ is determined by the identities

$$
X e_{n}^{\prime}=\sum_{m=1}^{\infty} x_{m n} e_{m}^{\prime \prime}
$$

Suppose that strongly continuous isometric representations $T_{k}: \mathbb{R} \rightarrow$ End $\mathscr{X}_{k}, k=1,2$, introduced by the identities

$$
T_{1}(t) x=\sum_{n=1}^{\infty} \mathrm{e}^{\mathrm{i} n t} \alpha_{n} e_{n}^{\prime}, \quad T_{2}(t) x=\sum_{n=1}^{\infty} \mathrm{e}^{\mathrm{i} n t} \beta_{n} e_{n}^{\prime \prime}
$$

are correct. Consider the bounded representation

$$
T: \mathbb{R} \rightarrow \text { End } \operatorname{Hom}\left(\mathscr{X}_{1}, \mathscr{X}_{2}\right), \quad T(t) X=T_{2}(t) X T_{1}(-t),
$$

where $x \in \mathscr{X}_{1}, f \in \mathrm{L}^{1}(\mathbb{R}), t \in \mathbb{R}, X \in \operatorname{Hom}\left(\mathscr{X}_{1}, \mathscr{X}_{2}\right)$, and the operator of the form

$$
(f X) x=\int_{\mathbb{R}} f(\tau) T_{2}(\tau) X T_{1}(-\tau) x \mathrm{~d} \tau .
$$

As $x=e_{n}^{\prime}$ we obtain the identities

$$
\begin{aligned}
(f X) e_{n}^{\prime} & =\int_{\mathbb{R}} f(\tau) T_{2}(\tau) X T_{1}(-\tau) e_{n}^{\prime} \mathrm{d} \tau=\int_{\mathbb{R}} f(\tau) T_{2}(\tau) X \mathrm{e}^{-\mathrm{i} n \tau} e_{n}^{\prime} \mathrm{d} \tau \\
& =\int_{\mathbb{R}} f(\tau) T_{2}(\tau) \mathrm{e}^{-\mathrm{i} n \tau} X e_{n}^{\prime} \mathrm{d} \tau=\int_{\mathbb{R}} f(\tau) T_{2}(\tau) \mathrm{e}^{-\mathrm{i} n \tau} \sum_{m=1}^{\infty} x_{m n} e_{m}^{\prime \prime} \mathrm{d} \tau \\
& =\sum_{m=1}^{\infty} \int_{\mathbb{R}} f(\tau) \mathrm{e}^{\mathrm{i}(m-n) \tau} x_{m n} e_{m}^{\prime \prime} \mathrm{d} \tau=\sum_{m=1}^{\infty} \widehat{f}(n-m) x_{m n} e_{m}^{\prime \prime}
\end{aligned}
$$


Thus, the matrix of operator $X$ reads as $\left(\widehat{f}(n-m) x_{m n}\right)$. Hence, $f X=0$ if and only if $\widehat{f}(n-m)=0$, $m, n \in \mathbb{N}$ for $x_{m n} \neq 0$, i.e., the Beurling spectrum of operator $X$ reads as

$$
\Lambda(X)=\left\{m, n \in \mathbb{N} \mid \text { there exists } f \in \mathrm{L}^{1}(\mathbb{R}) \text { such that } \widehat{f}(n-m)=0 \text { and } x_{m n} \neq 0\right\} .
$$

\section{BIBLIOGRAPHY}

1. N.I. Akhiezer. Lectures on approximation theory. Nauka, Moscow, 1965. (in Russian.)

2. Yu.I. Lyubich. Completeness conditions for a system of eigenvectors of a correct operator // Uspekhi Matem. Nauk. 1963. V. 18, No. 1. P. 165-171. (in Russian.)

3. A.G. Baskakov. Representation theory for Banach algebras, Abelian groups, and semigroups in the spectral analysis of linear operators // Sovrem. Matem. Funkts. Analiz. 2004. V. 9. P. 3-151. [ J. Math. Sci. 2006. V. 137, No. 4. P. 4885-5036.]

4. A.G. Baskakov. Harmonic analysis of linear operators. Voronezh State University, Voronezh, 1987. (in Russian.)

5. A.G. Baskakov. Inequalities of bernshtein type in abstract harmonic analysis // Sibir. Matem. Zhurn. 1979. V. 20, No. 5. P. 942-952. [Sib. Math. J. 1979. V. 20. No. 5. P. 665-672.]

6. N. Dunford, J.T. Schwartz. Linear Operators. Part 1. General Theory. Wiley-Interscience, New York, 1988.

7. N.S. Kaluzhin. Slowly varying functions and their behaviours // Vestnik VGU. Ser. Fiz. Matem. 2010. No. 2. P. 97-102. (in Russian.)

8. A.G. Baskakov. Spectral analysis of perturbed nonquasianalytic and spectral operators // Izv. RAN. Ser. matem. 1994. V. 58, No. 4. P. 3-320. [Russ. Acad. Sci. Izv. Math. 1995. V. 45, No. 1. P. 1-31.]

9. A.G. Baskakov, I.A. Krishtal. Harmonic analysis of causal operators and their spectral properties // Izv. RAN. Ser. matem. 2005. V. 69, No. 3. P. 3-54. [Izv. Math. 2005. V. 69, No. 3. P. 439-486.]

10. A.G. Baskakov, N.S. Kaluzhina. Beurling's theorem for functions with essential spectrum from homogeneous spaces and stabilization of solutions of parabolic equations // Matem. Zametki. 2012. v. 92, No. 5. P. 643-661. [Math. Notes. 2012. V. 92, No. 5-6. P. 587-605.]

Egor Evgen'evich Dikarev, Voronezh State University, Universitetskaya sq., 1 , 394006, Voronezh, Russia

E-mail: heiligenkreuz@gmail.com 